\title{
Therapieadhärenz bei Asthma bronchiale - Versorgung unter Alltagsbedingungen in der pneumologischen Praxis
}

\author{
Therapy Adherence in Bronchial Asthma Patients: \\ Healthcare under Everyday Conditions in Pulmonological Practices
}

\author{
Autoren \\ J. Andres ${ }^{1}$, A. Hellmann², T. Hering ${ }^{3}$, R. Hövelmann ${ }^{4}$, M. Weber ${ }^{5}$ \\ Institute \\ Med wiss GmbH, Weinitzen bei Graz \\ Pneumologe, Augsburg \\ 3 Pneumologe, Berlin \\ ${ }^{4}$ Chiesi GmbH, Hamburg \\ Pneumologe, Starnberg
}

eingereicht 14.1.2014 akzeptiert nach Revision 13.2.2014

\section{Bibliografie}

Dol http://dx.doi.org/ 10.1055/s-0034-1365285

Online-Publikation: 21.3.2014

Pneumologie 2014; 68: 315-321

(c) Georg Thieme Verlag KG

Stuttgart · New York

ISSN 0934-8387

\section{Korrespondenzadresse}

Jörg Andres

Med wiss $\mathrm{GmbH}$

Schöckelblickstraße 36

A-8044 Weinitzen bei Graz

j.andres@gmx.at

\section{Zusammenfassung \\ $\nabla$}

In der vorliegenden Untersuchung wurden Patienten mit Asthma bronchiale hinsichtlich ihrer Therapieadhärenz befragt. Es konnte gezeigt werden, dass die Adhärenz maßgeblich vom Alter, der Berufstätigkeit und der Qualität der Information zu und dem Interesse des Patienten an Erkrankung, Wirkweise und Handhabung des Inhalationsdevice beeinflusst wird. Jüngere, berufstätige Patienten, die sich nicht „völlig ausreichend“ informiert fühlen, sind eher nicht therapieadhärent. Dabei ist jedes Merkmal einzeln zu beachten.

Die Charakterisierung ist in der pneumologischen Praxis hilfreich bei der Bildung von Zielgruppen für geeignete Beratungsinitiativen und ermöglicht eine Kontrolle des Effektes der Maßnahme. Die Ergebnisse bestätigen die große Bedeutung der Arzt/Patienten Kommunikation für die Erreichung einer hohen Adhärenz und damit den Therapieerfolg.

Es wird vorgeschlagen, Asthma-Patienten folgende Fragen in der routinemäßigen Therapiekontrolle schriftlich - etwa als Ergänzung von ohnehin bereits verwendeten Fragebogen - vorzulegen, um aus dem Befragungsergebnis den $\mathrm{Zu}$ schnitt und das Ausmaß der notwenigen Beratung abzuleiten:

- Fühlen Sie sich zu Ihrer Erkrankung ausreichend informiert?

- Fühlen Sie sich zur Wirkweise der Arzneimitteltherapie ausreichend informiert?

- Fühlen Sie sich zur Handhabung der Inhalationsmedikamente ausreichend informiert?

- Sind Sie mit der Handhabung Ihres Inhalationsmedikamentes zufrieden?

Die Antwortmöglichkeiten je Frage sollten lauten: völlig - ziemlich - weniger - überhaupt nicht.

Es zeigten sich deutliche Unterschiede hinsichtlich der Adhärenz zwischen Patienten, die mit der Handhabung ihres Device völlig zufrieden

\section{Abstract \\ $\nabla$}

In the present study, patients with asthma were interviewed with regard to their treatment adherence. It was shown that adherence is significantly influenced by age, occupation, quality of information about the disease and interest of the patient in disease, mode of action and use of the inhalation device. Younger, working patients who do not feel "completely adequately" informed are more likely to be not-adherent. Each aspect should be noted separately.

The characterization enables forming target groups for appropriate counselling initiatives in pulmonology practice and allows the effectiveness of the measures to be examined. The results confirm the importance of doctor/patient communication for achieving a high level of adherence and thus therapeutic success.

It is suggested that, in addition to questionnaires already in use, asthma patients should be given the following questions in writing during routine monitoring of therapy; based on the results of the survey, the extent of advice necessary can be determined

- Do you feel sufficiently informed about your illness?

- Do you feel sufficiently informed about the effect of the drug therapy?

- Do you feel sufficiently informed about the handling of the inhalationdrugs?

- Are you satisfied with the handling of your inhalation medication?

The following options to answer the questions should be available:

completely - mainly - somewhat - not at all

There were significant differences in adherence between patients who were completely satisfied with the handling of their device and those that were not. However, there was no significant difference between the devices. Therefore, the same therapy adherence can be predicted for all devices 
waren, und jenen, die das nicht waren. Jedoch fand sich kein signifikanter Unterschied zwischen den Devices. Bei patientengerechter Deviceauswahl, die auch völlige Zufriedenheit mit der Handhabung zum Ziel hat, ist bei jedem Device von einer vergleichbaren Therapieadhärenz auszugehen.

Da es sich um subjektive Wahrnehmungen der Patienten handelt, zeigen die Daten erhebliche Unterschiede zwischen den Studienzentren. Diese wurden in einem verdeckten Benchmark zurück gespiegelt, um Optimierungen anzuregen.

\section{Einleitung}

Die WHO definiert Therapieadhärenz als „Ausmaß, in dem der Patient den medizinischen Anweisungen folgt“ [1]. Bei Asthma bronchiale wird als wichtigstes Element der Therapieadhärenz die regelmäßige Anwendung der Dauertherapie nach Verordnung gesehen [2].

Asthma bronchiale ist eine chronisch entzündliche Krankheit. Die Asthma-Kontrolle hängt maßgeblich von der konsequenten antientzündlichen Therapie ab. Für Patienten mit dauerhafter Notwendigkeit einer Therapie mit inhalativen Steroiden gilt: Die Krankheit exacerbiert häufiger, wenn die Behandlung nicht kontinuierlich durchgeführt oder sogar unterbrochen wird [3]. Die Behandlungskosten sind bei mangelnder Adhärenz zumindest bei schwerem Asthma höher [4].

Maßgebliche Leitlinien wie die nationale Versorgungsleitlinie [5] fordern die kontinuierliche antiinflammatorische Therapie ab Schweregrad II. Inhalative Steroide sind nicht mit einer adhärenzverstärkenden Akutwirkung ausgestattet. Deshalb ist hier das Erreichen einer guten Adhärenz besonders schwierig, aber wichtig. Therapietreue (Adhärenz) ist somit der Schlüssel zum Erfolg einer Asthmatherapie. Bisherige Untersuchungen zur Adhärenz zeigen, dass hier erhebliche Defizite bestehen. Maximal $50 \%$ der Patienten sind als therapieadhärent anzusehen [6]. Ein wesentliches Ziel der Behandlungsstrategie einer pneumologischen Praxis muss es deshalb sein, die Therapieadhärenz zu verbessern. Dabei wären Hinweise hilfreich, die Patienten herauszufiltern, die besondere Impulse benötigen, um therapieadhärent zu sein. Weiter wäre es nützlich, im Routinebetrieb leicht zu gewinnende Indikatoren zu identifizieren, die darauf schließen lassen, ob gesetzte Impulse tatsächlich eine verbesserte Therapieadhärenz erwarten lassen.

\section{Material und Methoden}

Zur Bestimmung der Therapieadhärenz werden in der Literatur verschiedene Methoden benutzt. Alle bieten jedoch nur ein ungefähres Abbild der Realität [7]. In der vorliegenden Untersuchung aus dem Jahr 2012 wurde die schriftliche Befragung des Patienten gewählt, weil es sich um eine einfache, in der täglichen Praxis einsetzbare Methode handelt, die Therapieadhärenz zu erfassen. Zusätzlich machte die Praxis auf einem separaten Dokumentationsbogen weitere Angaben z. B. zur aktuellen Therapie. Vorgaben zur Art der Therapie gab es nicht.

Jede der insgesamt 52 pneumologischen Praxen erhielt 100 Fragebogen. Ab Studienbeginn wurden diese an jeden Patienten mit Asthma bronchiale ausgegeben, der den folgenden Einschlusskriterien entsprach: when the device is suitable for the patient and also provides complete satisfaction in handling.

Since it is the subjective perception of patients, the data show significant differences between study centres. These were mirrored in a blind benchmark to stimulate improvements.

- Diagnose Asthma bronchiale ist seit mindestens 3 Monaten gesichert,

- die Erkrankung wird seit mindestens 3 Monaten medikamentös behandelt,

- Mindestalter 18 Jahre.

War ein Patient nicht bereit, den Fragebogen auszufüllen, wurde das auf einem besonderen Bogen dokumentiert. 40 Studienzentren schlossen mindestens 60 Patienten in die Untersuchung ein und erhielten eine auf das jeweilige Zentrum bezogene Auswertung.

Die Patienten bearbeiteten ihre Fragen ohne Supervision durch das Praxisteam. Sie wurden informiert, dass ihre Angaben völlig anonym ausgewertet werden. Den Fragebogen deponierten sie in einem geschlossenen Briefkasten. Ziel war es, möglichst unbeeinflusste Antworten der Patienten zu erhalten.

Im Fragebogen waren drei Fragen zur Therapieadhärenz hinsichtlich der konsequenten Durchführung der erforderlichen Basistherapie mit vorgegebenen Antwortoptionen enthalten:

- Wie oft haben Sie in den letzten 4 Wochen die Anwendung Ihres Basismedikamentes der Asthmatherapie vergessen? (Nie $=$ Indiz für gute Adhärenz)

- Haben Sie in den letzten 12 Monaten die Einnahme Ihres zur regelmäßigen Anwendung verordneten Asthmamedikamentes aufgrund fehlender Beschwerden eigenständig für eine bestimmte Zeit ausgesetzt oder ganz abgesetzt?

(Nie= Indiz für gute Adhärenz)

- Haben Sie in den letzten 12 Monaten abweichend von der Vorgaben des Arztes die Häufigkeit der Anwendung Ihres Asthma-Medikamentes geändert?

(Nein = Indiz für gute Adhärenz)

Wenn ein Patient alle drei Fragen im Sinne der „guten“ Adhärenz beantwortet hat, wurde er als vollständig adhärent gewertet. Denn Ziel in der Praxis ist es, dass möglichst viele Patienten diese Gruppe erreichen.

\section{Ergebnisse}

Der Rücklauf betrug 3827 Fragebogen. Von diesen konnten hinsichtlich der Therapieadhärenz 3293 (86\%) ausgewertet werden, da alle drei Fragen zur Therapieadhärenz eindeutig beantwortet wurden.

Davon waren $49 \%$ therapieadhärent, $51 \%$ waren nicht vollständig adhärent. Die nicht vollständig adhärenten waren wie folgt abgestuft: $30 \%$ waren in einer Frage nicht adhärent, $13 \%$ in zwei Fragen und 7\% in keiner Frage (s. $\odot$ Abb.1).

Die Altersstruktur der Stichprobe stimmt weitgehend mit der erwarteten Altersverteilung in einer pneumologischen Praxis überein. Das zeigt der Vergleich mit einer eigenen quantitativen Erhebung in 36 pneumologischen Praxen mit 69 Pneumologen und 31681 Asthmapatienten (Publikation des BdP in Vorbereitung). 


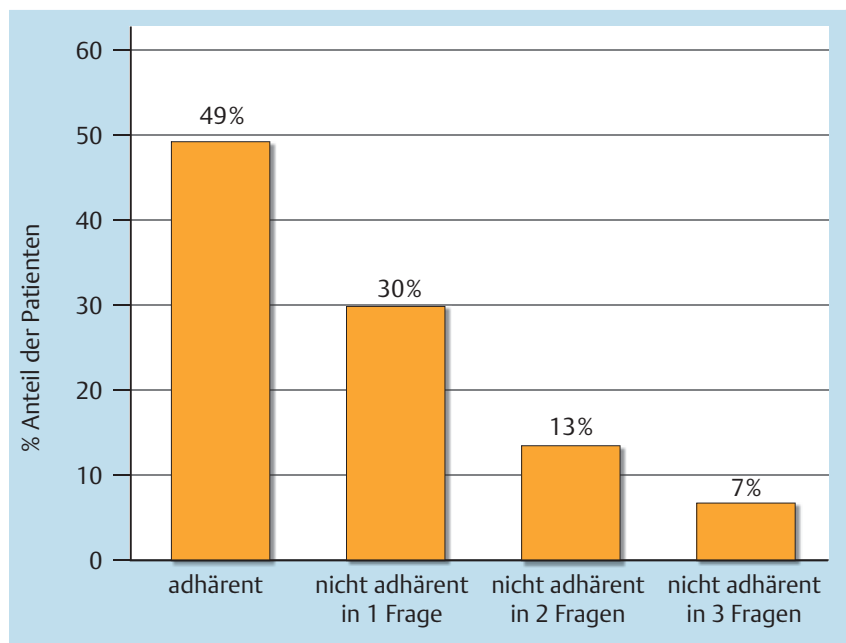

Abb.1 Verteilung adhärenter und in 3 Graduierungen nicht adhärenter Patienten. Korrelationskoeffizient 0,98.

Der Anteil der erwachsenen adhärenten Patienten steigt mit dem Alter ( $\bullet$ Abb.2). 39\% der Patienten bis 49 Jahre sind adhärent. Bei der Gruppe ab 50 Jahre und älter steigt der Anteil der adhärenten auf 59\% $(\mathrm{p}<0,001)(\bullet$ Abb. 3$)$.

Ein ähnliches Verhältnis zeigt sich auch hinsichtlich der Berufstätigkeit. $41 \%$ der berufstätigen Patienten sind adhärent. Bei den nicht Berufstätigen beträgt dieser Anteil $61 \%(p<0,001)$. Alter und Berufstätigkeit sind natürlich keine unabhängigen Merkmale.

Die Patienten wurden nach ihrer subjektiven Zufriedenheit mit der Information zur Erkrankung bei der Erstdiagnose befragt. $40 \%$ gaben an, damit völlig zufrieden gewesen zu sein. $41 \%$ waren ziemlich, $12 \%$ weniger zufrieden, und $7 \%$ empfanden die Information als nicht ausreichend. Obgleich die Asthmaerkrankung bei $93 \%$ der Patienten bereits länger als 12 Monte besteht und damit die Erstinformation auch zeitlich länger zurückliegt, zeigt sich ein Effekt auf die Therapieadhärenz. 57\% der Patienten, die mit der Erstinformation „völlig zufrieden“ waren, sind therapieadhärent. Bei den anderen Patienten sind nur $44 \%$ adhärent $(\mathrm{P}<0,001)$.

Eine Mehrheit der Patienten (69\%) hält eine Aufklärung zur Wirkweise der verordneten Medikamente für „sehr wichtig“. „Völlig zufrieden“ mit der Aufklärung zur Wirkweise waren aber nur 56\% der Asthmatiker.
$53 \%$ der Patienten, denen eine Information über die Wirkweise der Medikamente „sehr wichtig“ ist, sind adhärent. Bei den Patienten, denen diese Information „ziemlich“, „weniger“ oder „überhaupt nicht" wichtig war, beträgt der Anteil der Adhärenten $40 \%(\mathrm{p}<0,001)$ (s. $\odot$ Abb. 4$)$.

Die Gruppe derjenigen, die subjektiv „völlig zufrieden“ mit der Information zur Wirkweise ihres Medikamentes waren, waren zu 56\% adhärent. Diejenigen, die sich „ziemlich“, „weniger“ oder „nicht ausreichend“ informiert fühlten, waren nur zu $40 \%$ adhärent $(\mathbf{p}<0,001)$ (s. $\odot$ Abb.4).

Hinsichtlich der Einschätzung der Information zur Wirkweise des Asthmamedikamentes fühlten sich $55 \%$,völlig ausreichend“ informiert. Hinsichtlich der Handhabung des Asthmadevice betrug dieser Wert 76\%, 69\% war eine gründliche Information zur Wirkweise „sehr wichtig“. Der entsprechende Wert zur Handhabung lag bei 78\%. Das Interesse an der Handhabung ist somit größer als das Interesse an der Wirkweise ( $\bullet$ Abb.5).

Wie bei den Patienten, denen die Information zur Wirkweise "sehr wichtig“ war, ist der Anteil der adhärenten Patienten mit $51 \%$ höher als bei denjenigen mit anderen Nennungen. Bei den Nennungen „ziemlich“, „,weniger“ oder „überhaupt nicht“ wichtig betrug der Anteil 40\% (p<0,001) (s. $\odot$ Abb.4). Die hinsichtlich der Anwendung ihres Medikamentes subjektiv „völlig ausreichend" informierten Patienten waren zu 52\% adhärent. Diejenigen, die sich „ziemlich“, „weniger“ oder "nicht ausreichend“ informiert fühlten, waren wie bei der Information zur Wirkweise nur zu $40 \%$ adhärent $(\mathrm{p}<0,001)$ (s. ๑ Abb.4).

Die Patienten wurden mit Hilfe von Piktogrammen befragt, welches Device sie aktuell nutzen und wie zufrieden sie damit sind. $22 \%$ der hinsichtlich der Therapieadhärenz auswertbaren Rückläufe machten dazu keine Angaben. 53\% benannten genau ein Device, $21 \%$ zwei und 3\% mehr als zwei Devices.

Am häufigsten wurde mit $51 \%$ das Spray genannt, gefolgt von Turbohaler (24\%), Diskus (21\%) und Novolizer (20\%). Der Autohaler war mit $14 \%$ etwas seltener. Aerolizer und Respimat waren mit 5\% und 3\% so selten, dass diese Devices in der Auswertung hinsichtlich Zufriedenheit und Adhärenz nicht weiterverfolgt werden konnten (s. $\odot$ Abb.6).

Bei der mit Piktogrammen gestützten Frage nach den derzeit genutzten Devices wurde nicht nach Bedarfs- und Dauertherapie unterschieden. Die Bedeutung einer Bedarfsmedikation wird häufig überschätzt. In der Versorgungsrealität haben Asthmapatienten oft keine Beschwerden und sehen daher keine Not-

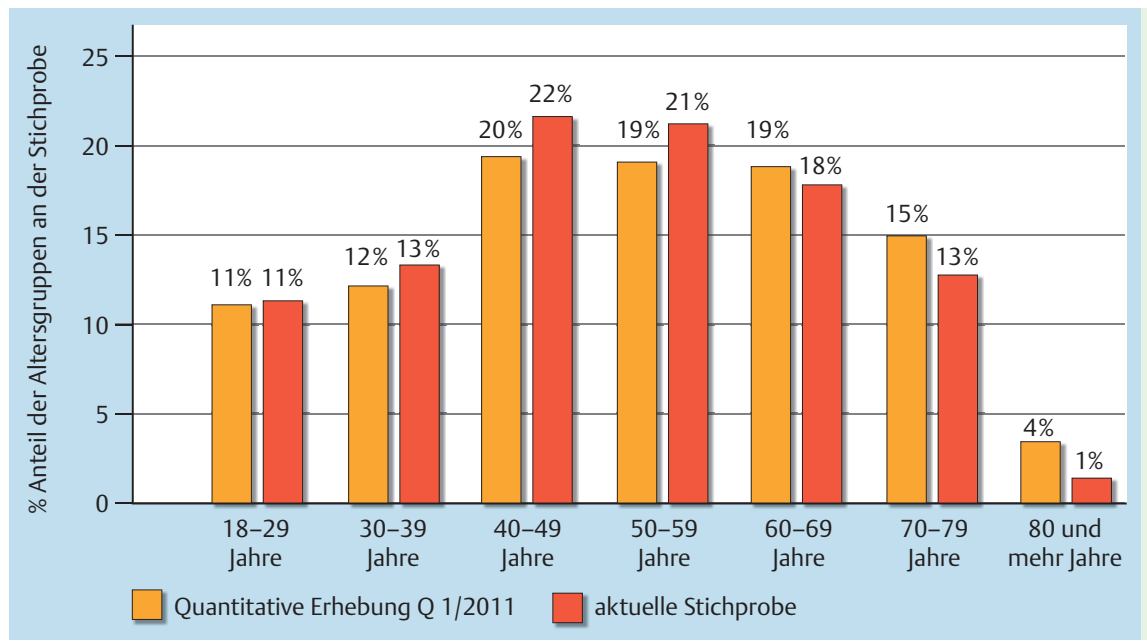

Abb. 2 Altersverteilung Asthmapatienten Vollerhebung versus Stichprobe. 


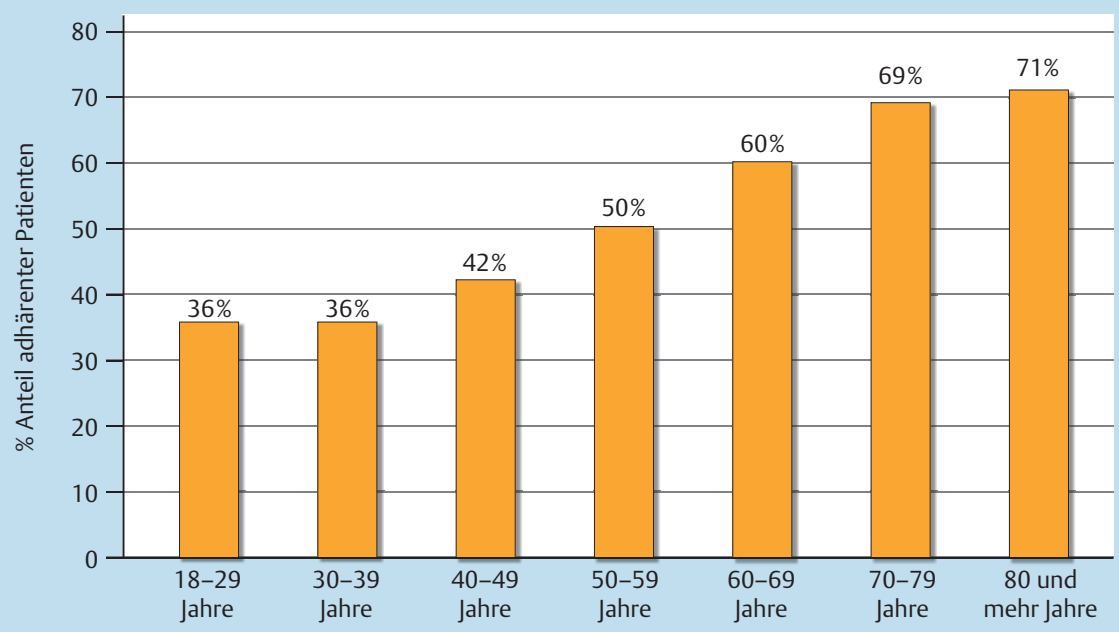

Abb.3 Anteil der adhärenten Patienten in den verschiedenen Altersgruppen.

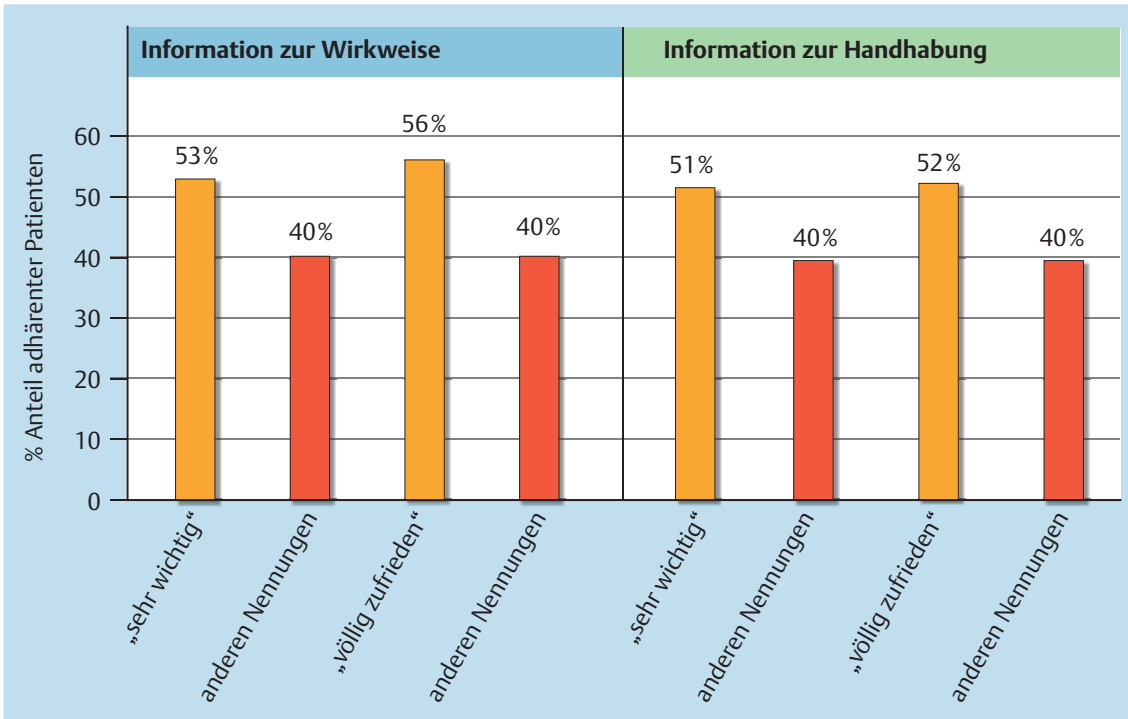

Abb.4 Anteil der adhärenten Patienten an den Gruppen, denen die Information zur Wirkweise/ Handhabung ihres Medikamentes „sehr wichtig“ war versus anderen Nennungen und Anteil der adhärenten Patienten, die mit der Information zur Wirkweise/Handhabung „sehr zufrieden“ waren.

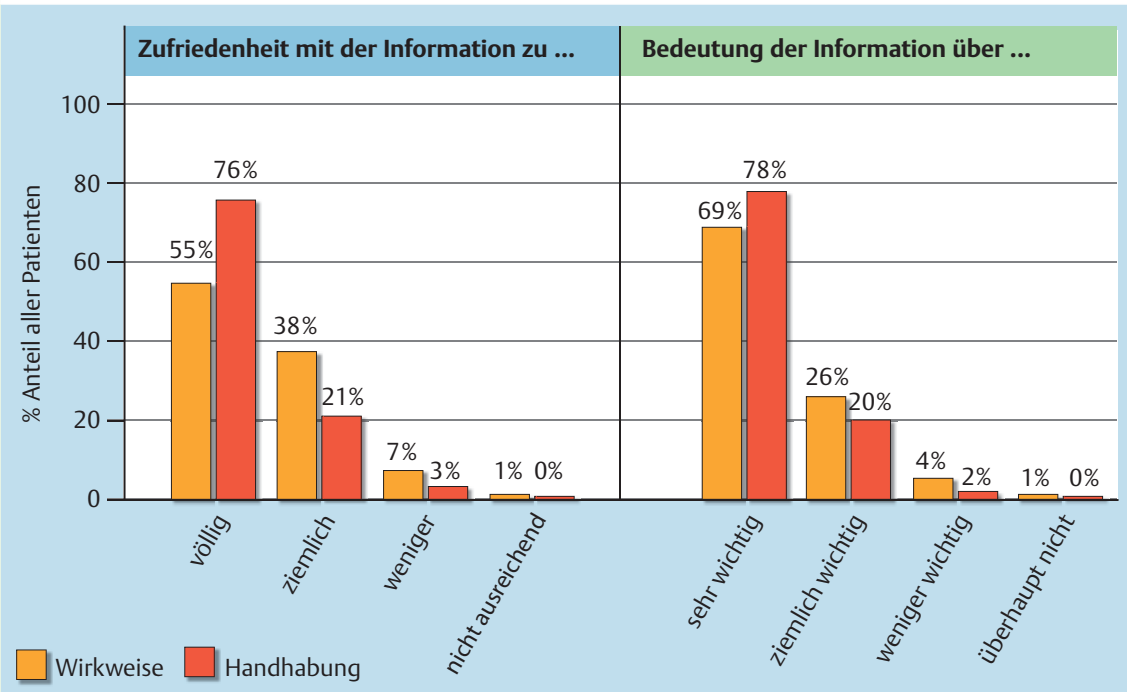

Abb.5 Übersicht über die Zufriedenheit aller Patienten mit der Information hinsichtlich Wirkweise und Handhabung ihres Medikamentes (linke Säulen) und hinsichtlich der Bedeutung, die sie dieser Information beimessen (rechte Säulen). 


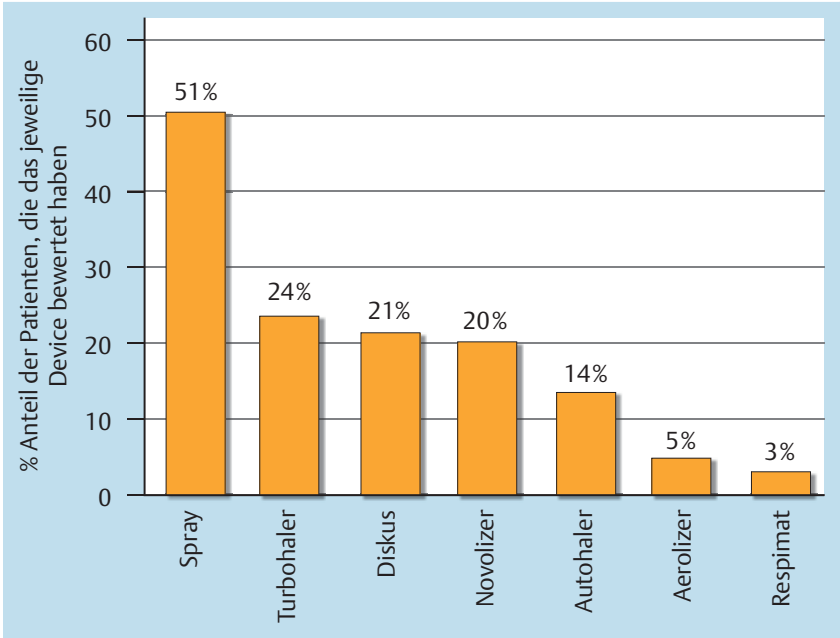

Abb.6 Anteil der Patienten, die das jeweilige Device derzeit nutzen.

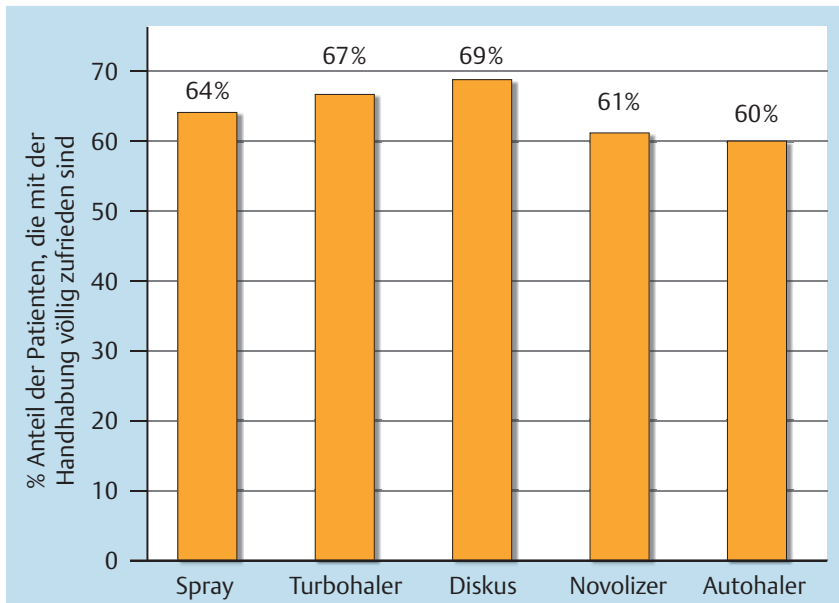

Abb.7 Anteil der Patienten, die mit der Handhabung des jeweiligen Device „völlig zufrieden“ sind.

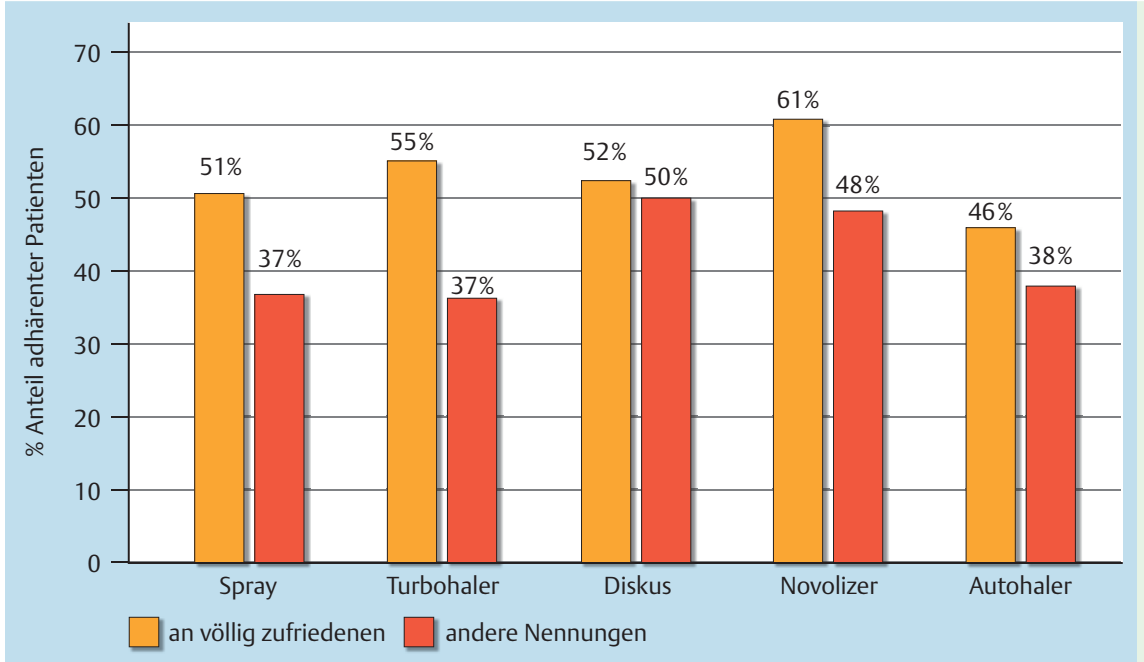

Abb.8 Anteil adhärenter Patienten je Device an mit der Handhabung „völlig zufriedenen“ Patienten versus denjenigen, die nicht völlig zufrieden waren.

wendigkeit für eine Bedarfsmedikation. Das ist das Resultat einer effektiven Dauertherapie.

Ein grundsätzlicher Unterschied in der Zufriedenheit mit der Handhabung unterschiedlicher Devices zeigte sich nicht. Die Anteile der „völlig Zufriedenen“ bei den verschiedenen Devices lagen zwischen $60 \%$ und 69\% (nicht signifikant) (s. Abb.7).

Über alle Devices betrachtet, waren 51\% der Patienten adhärent, die mit der Handhabung des Device völlig zufrieden waren. 38\% derjenigen waren adhärent, die ziemlich, weniger oder überhaupt nicht zufrieden waren ( $\mathrm{p}<0,001)$.

Bezogen auf die einzelnen Devices zeigte sich der gleiche Trend ( $\bullet$ Abb. 8). Der Anteil der Adhärenten lag bei den „Völlig zufriedenen“ jeweils höher als bei den anderen. Bei den „völlig zufriedenen“ lagen die Anteile der adhärenten Patienten zwischen 46\% und $61 \%$. Bei den Patienten, die nicht „völlig zufrieden“ waren, lagen die Anteile zwischen 37\% und 50\% ( Abb.8).

Die Unterschiede hinsichtlich der Adhärenzwerte zwischen den Devices sind nicht signifikant.

ACT, FEV1 und Therapieadhärenz korrelierten nicht signifikant! Ein Zusammenhang war auch nicht zu erwarten, da die Untersuchung als Endpunkt-Untersuchung keine Verläufe erfassen kann. Denn Patienten wurden in unterschiedlichen Phasen ihrer Erkrankung in die Untersuchung aufgenommen. Nur bei einer Er- hebung im Zeitverlauf wäre eine Korrelation denkbar. Zusätzlich müsste der Schweregrad der Erkrankung vor Therapie bestimmt werden. Denn schwerere Erkrankungen mit effektiver Therapie führen zum gleichen Kontrollgrad wie leichtere Verläufe mit mangelnder Adhärenz. Diese Parameter waren aber nicht Gegenstand der Untersuchung.

Für Studienzentren, die mindestens 60 Fälle beigesteuert haben, wurde eine praxisbezogene Auswertung erstellt. Dabei zeigten sich deutliche Unterschiede von Zentrum zu Zentrum bei der Beantwortung der Fragen zur Therapieadhärenz ( $\bullet$ Abb.8). Der erreichte Rang zwischen den Studienzentren betrug $44 \%$ und $75 \%$ bei der Frage nach der Medikamenteneinnahme. Bei der Frage zur Unterbrechung der Therapie ohne ärztlichen Rat lagen die Unterschiede zwischen 53\% und 93\%. Zwischen 59\% und $91 \%$ lagen die Unterschiede bei der Frage, ob die Dosierung eigenmächtig ohne ärztliche Anweisung geändert wurde.

Auch hinsichtlich der subjektiven Beurteilung der Information zu Wirkweise und Handhabung des Asthmamedikamentes gab es deutliche Unterschiede von Studienzentrum zu Studienzentrum. Sieht man von den Werten der laufenden Nummer $1 \mathrm{ab}$, waren zwischen $42 \%$ und $76 \%$ mit der Information zur Wirkweise ihres Asthmamedikamentes „völlig zufrieden“, mit der Information zur 


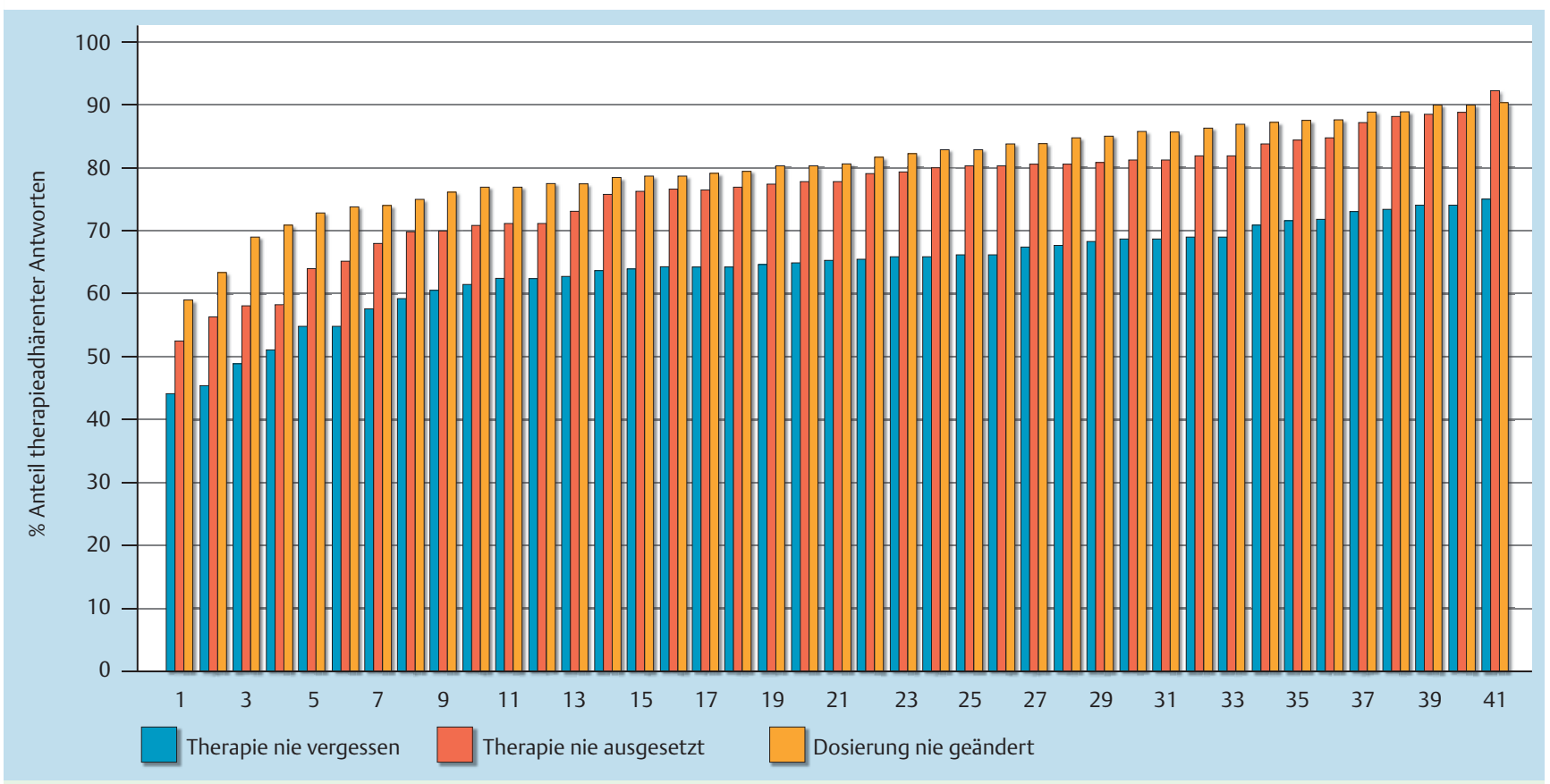

Abb.9 Anteil der Patienten, die nie die Therapie vergessen oder vorsätzlich ausgesetzt oder die Dosierung geändert haben. Werte je Praxis.

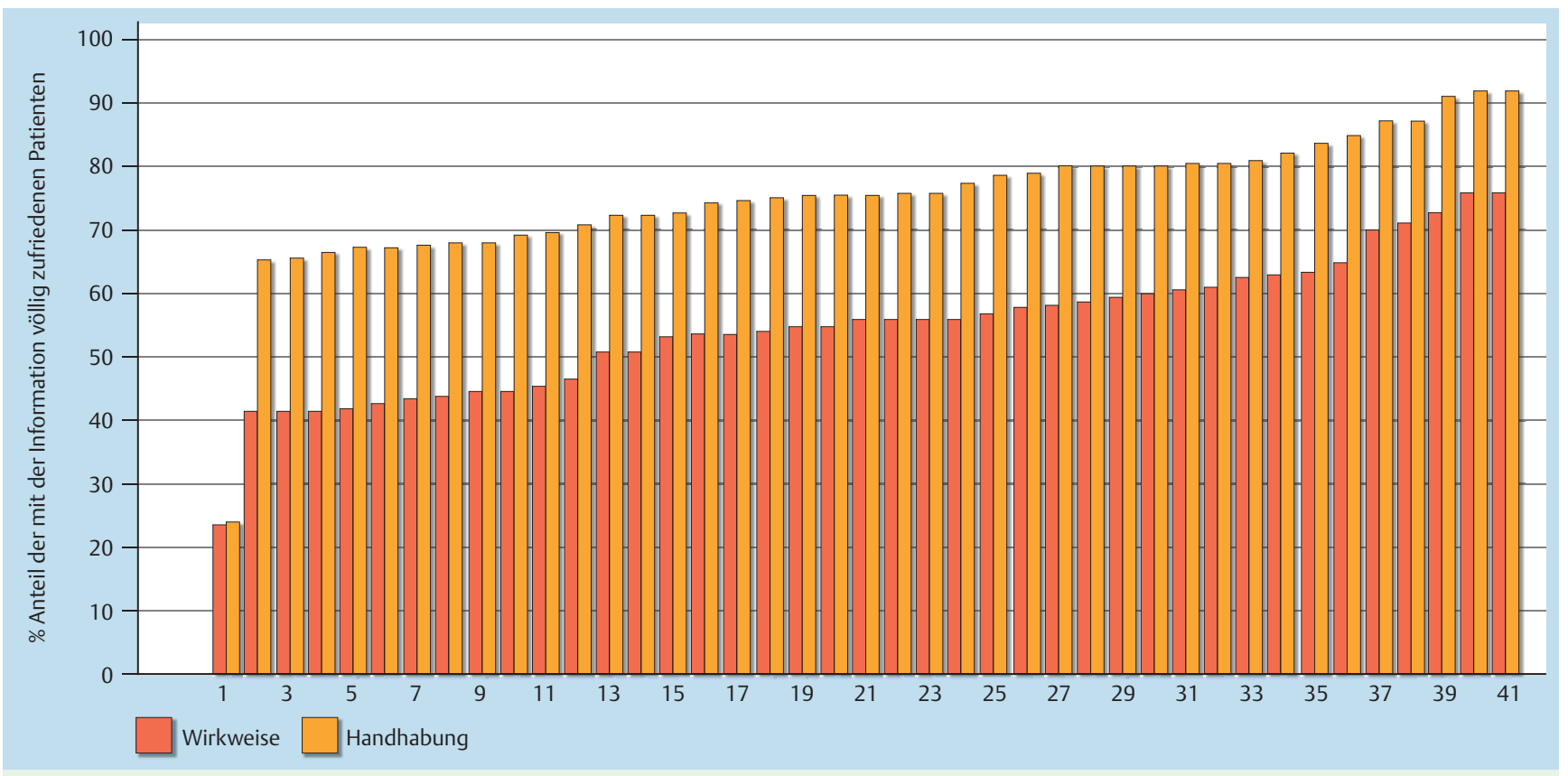

Abb.10 Anteil der Patienten, die mit der Information zu Wirkweise und Handhabung des verordneten Medikamentes „völlig zufrieden“ waren. Werte je Praxis.

Handhabung waren es zwischen $66 \%$ und $92 \%$ Abb.9 und - Abb. 10).

Eine Korrelation der Adhärenz mit der Zufriedenheit der Information auf Praxisebene ist wegen der geringen Fallzahl/Praxis nicht sinnvoll.

In der Untersuchung wurden die erhobenen Ergebnisse an die teilnehmenden Praxen zurückgespielt (verdecktes Benchmark). Nur die eigenen Praxisdaten wurden offenbart (farblich abgesetzt), das Kollektiv bleibt anonym.

\section{Diskussion}

Therapieadhärenz ist die Voraussetzung für eine leitliniengerechte Behandlung des Asthmas. Gerade bei einer Erkrankung mit einer großen Variabilität ist die regelmäßige Durchführung einer inhalativen Dauertherapie eine große Herausforderung. In der pneumologischen Praxis wird man sich bei jedem Patienten bemühen, eine optimale Therapieadhärenz zu erreichen. Die erhobenen Daten ermöglichen es, diejenigen Patienten zu charakterisieren, bei denen ein geringerer Anteil therapieadhärenter zu erwarten ist. 
In der vorgelegten Untersuchung konnten dafür folgende Merkmale identifiziert werden:

- Lebensalter bis 49 Jahre oder

- Berufstätigkeit oder

- mangelnde Aufklärung zur Erkrankung bei Erstdiagnose oder

- mangelndes Interesse/mangelnde Zufriedenheit des Patienten an/mit der Information zur Wirkweise der Therapie oder

- mangelndes Interesse/mangelnde Zufriedenheit des Patienten an/mit der Information zur Handhabung des Device oder

- mangelnde Zufriedenheit mit der Handhabung des Device.

Patienten, die diesen Gruppen angehören, sollte hinsichtlich der Optimierung der Therapieadhärenz besondere Aufmerksamkeit gewidmet werden.

Lebensalter und Berufstätigkeit sind in der Praxis bekannt.

Die folgenden Parameter sind über den routinemäßig eingesetzten Patienten-Fragebogen leicht zu erheben. Dazu werden folgende vereinfachte Fragen vorgeschlagen, die in einen etwaig schon bestehenden Bogen integriert werden können:

- Fühlen Sie sich zu Ihrer Erkrankung ausreichend informiert?

- Fühlen Sie sich zur Wirkweise der Arzneimitteltherapie ausreichend informiert?

- Fühlen Sie sich zur Handhabung der Inhalationsmedikamente ausreichend informiert?

- Sind Sie mit der Handhabung Ihres Inhalationsmedikamentes zufrieden?

In der vorliegenden Untersuchung hat sich eine abgestufte Antwortskala mit den Werten „völlig“, „ziemlich“, „weniger“ oder „nicht ausreichend“ bewährt. Falls der Patient nicht „völlig ausreichend“ informiert bzw. „Völlig zufrieden“ ist, kann von einer erhöhten Wahrscheinlichkeit ausgegangen werden, dass er nicht adhärent ist.

Es bietet sich an, solchermaßen als eher nichtadhärent identifizierten Patienten besondere Aufmerksamkeit zu widmen. Bei mangelndem Interesse für die eigene Krankheit und die zugehörige Therapie gehört hoher Motivationsaufwand und in jedem Fall genügend Information über Erkrankung, Wirkweise der Therapie und Handhabung des verordneten Device dazu, um die Adhärenz anzuheben. Die so identifizierten Patienten würden besonders von einer strukturierten Schulung profitieren.

Auch zur Kontrolle des Erfolges einer gesteigerten Aufklärung und Information bietet sich die einfache schriftliche Befragung des Patienten an. Bei regelmäßiger Anwendung des Fragebogens kann ein Wechsel der Patientenwertung dokumentiert werden. Es gab keine Hinweise auf eine unterschiedliche Zufriedenheit der Patienten mit einem bestimmten Device. Die patientengerechte Auswahl und Einweisung in die Handhabung des Inhalators ist somit wesentlicher für die Adhärenz als eine bestimmte Technologie bei der Handhabung. In der Untersuchung wurden die praxisindividuellen Daten an die teilnehmenden Studienzentren zurückgespielt. Dadurch hat die ärztliche Leitung die Möglichkeit, aus der Interpretation der praxisbezogenen Daten Kon- sequenzen zu erarbeiten. Ob der Praxisvergleich tatsächlich zu einer Steigerung der Versorgungsqualität führt, ist aber weiteren Untersuchungen vorbehalten.

Stärken und Schwächen der Untersuchung: Zur Ermittlung der Therapieadhärenz wurden Patientenangaben herangezogen. Man kann wohl davon ausgehen, dass dadurch die Therapieadhärenz eher zu optimistisch abgebildet wird. Denn auch wenn die anonyme Abgabe des Dokumentationsbogens gewährleistet war, können Gefälligkeitsangaben nicht völlig ausgeschlossen werden. Im Alltag der pneumologischen Praxis ist man jedoch genau auf diese Angaben angewiesen, da pneumologische Patienten häufig von Haus- und Facharzt gemeinsam versorgt werden. Daher sind die Informationen zum Krankheitsverlauf und zur Arzneimitteltherapie nicht quantitativ an einer Stelle verfügbar.

Die Fragen, die benutzt wurden, um Patienten hinsichtlich ihrer Therapieadhärenz zu charakterisieren, sind nicht evaluiert. Sie sind aus dem pneumologischen Arztgespräch abgeleitet und sind alltagstauglich.

\section{Danksagung}

Wir danken der Chiesi GmbH für die ideelle und finanzielle Unterstützung bei der Durchführung dieser Untersuchung.

\section{Interessenkonflikt}

$\nabla$

J. Andres, A. Hellmann, T. Hering, M. Weber geben an, dass kein Interessenkonflikt besteht.

R. Hövelmann ist Mitarbeiter der Chiesi GmbH, Hamburg.

\section{Literatur}

1 Sabaté E. Adherence to long-term therapies. Evidence of action. World Health Organization. 2003: 1-194

2 Fischer J, Wimmer A, Mahlich J. Medikamentenadhärenz in der Asthmatherapie - eine strukturierte Übersicht. Pneumologie 2013; 67: $406-414$

3 Hagiwara M, Delea TE, Stanford RHI. Risk of asthma exacerbation, asthma-related health care utilization and costs, and adherence to controller therapy in patients with asthma receiving fluticasone propionate/ salmeterol inhalation powder $100 \mathrm{mug} / 50$ mug versus mometasone furoate inhalation powder. J Asthma 2013; 50: 287-295

4 Mäkelä MJ, Backer V, Hedegaard M et al. Adherence to inhaled therapies, health outcomes and costs in patients with asthma and COPD. Respir Med 2013; 107: 1481 - 1490

5 Nationale Versorgungs Leitlinie Asthma. 2. Auflage. http://www.versorgungsleitlinien.de

6 Gillissen A. Patients' adherence in asthma. J Physiol Pharmacol 2007; 58 (Suppl. 05): 205-222

7 Gillissen $A$ et al. Therapieadhärenz bei Asthma bronchiale. Dtsch med. Wochenschr 2007; 132: 1281-1286 